\title{
Evaluación financiera de la pyme del sector construcción de vivienda en Colombia (2011-2016)*
}

\section{Financial evaluation of the SME of the homebuilding sector in Colombia (2011-2016)}

\author{
Jorge Alberto Rivera Godoy**
}

Recibido: 15 de enero de 2019

Revisado: 30 de marzo de 2019

Aprobado: 10 de junio de 2019

\footnotetext{
Este artículo es producto de uno de los proyectos de investigación realizados por el Grupo de Investigación de la Universidad del Valle denominado Generación de Valor Económico, adscrito a línea sobre evaluación del desempeño financiero de empresas del sector real en Colombia. Cómo citar este artículo: Rivera Godoy, J. A. (2019). Evaluación financiera de la pyme del sector construcción de vivienda en Colombia (2011-2016). Revista CIFE: Lecturas de Economía Social, 21(35), 141-165. DOI: https://doi.org/10.15332/22484914.5373

* Doctor distinguido cum laude en Ciencias Económicas y Empresariales por la Universidad Autónoma de Madrid, España. Especialista en Finanzas de la Universidad del Valle, Cali, Colombia. Profesor titular de la Universidad del Valle, Facultad de Ciencias de la Administración, Departamento de Contabilidad y Finanzas. Coordinador del grupo de investigación en Generación de Valor Económico (GIGVE) de la Universidad del Valle (categoría C de Colciencias). Correo electrónico: jorge.rivera@correounivalle.edu.co Cali, Colombia. http://orcid. org/0000-0003-2319-1674
} 


\section{Resumen}

El documento muestra los principales hallazgos de un análisis que tiene como intención el de valorar el comportamiento financiero de la pyme del sector construcción de vivienda en Colombia en el período 2011-2016, siguiendo como metodología el estudio de algunos de los más importantes indicadores de desempeño contables y de valoración a nivel económico para determinar el grado de cumplimiento del objetivo financiero de las empresas. La pyme del sector de la construcción en Colombia logra rendimientos contables, aunque superiores en la mediana empresa en razón a su eficacia en el control de los gastos y al uso de un mayor apalancamiento financiero positivo. No obstante lo anterior, los resultados también demuestran que existe una destrucción del valor económico agregado (EVA) en los años analizados. Esta destrucción de valor obedece a que el costo de recursos financieros es superior a la utilidad operacional después de impuestos.

Palabras clave: economic value added (EVA), valor de mercado agregado (VMA), indicadores financieros de eficiencia, eficacia y efectividad, pyme de construcción de viviendas.

Códigos JeL: G10, G32, M40, L74.

\section{Abstract}

The document shows the main findings of an analysis that intends to assess the financial behavior of the SME in the homebuilding sector in Colombia in the period 2011-2016, following as a methodology the study of some of the most important performance indicators accounting and valuation at the economic level to determine the degree of compliance with the financial objective of the companies. The SME of the construction sector in Colombia achieves accounting returns, although higher in the medium-sized company due to its efficacy in controlling expenses and costs, and the use of greater positive financial leverage. Notwithstanding the foregoing, the results also show that there is a destruction of the economic added value (EvA) in the years analyzed. This destruction of value is due to the fact that the cost of financial resources is higher than the operating income after taxes.

Keywords: economic value added (EVA), market value added (VMA), financial indicators of efficiency, efficacy, and effectiveness, sME of the homebuilding sector.

Classification JEL: G10, G32, M40, L74. 


\section{Introducción}

El sector construcción de vivienda ha sido catalogado en los últimos años como uno de los pilares para el crecimiento de la economía y el empleo en Colombia, a tal punto que en el gobierno de Juan Manuel Santos se le consideró como una de las cinco locomotoras que impulsaría la generación de empleo y desarrollo de ciudades amables (DNP, 2017, p. 14). No obstante, los avances que ha tenido este sector al reducir el déficit de vivienda urbana y la generación de nuevos puestos de trabajo (Revista Semana, 2016), es poco lo que se sabe sobre su desempeño financiero, y en particular si la pyme de este sector, que para el año 2016 representaba el 55\%, es rentable y creadora de valor económico, y por qué; siendo esta información de gran interés para la comunidad académica, empresarial y estatal.

Para formalizar este artículo se ha recurrido al uso de la metodología del cálculo y estudio de los principales resultados de los indicadores contables que determinan el comportamiento en el tiempo de las principales medidas financieras de las empresas de uso común relacionadas con el comportamiento de dichas empresas en un momento determinado del tiempo (Ross, Westerfield, \& Jordan, 2014; Rivera, 2004; Martin, \& Petty, 2001; Milla, 2010).

Algunos de los principales hallazgos determinan que las ventas y el comportamiento de los activos presentan tendencias crecientes; se logran rentabilidades contables, que son superiores para la mediana empresa que muestra una mayor eficacia en el control de costos y gastos, y mayor uso de un apalancamiento financiero positivo, aunque es menos eficiente en el uso de los activos que la pequeña empresa. Estos resultados favorables son contrarios a los encontrados mediante los cálculos realizados para los indicadores de gestión. La pyme del sector construcción de vivienda para el caso colombiano se destruye EVA. Los resultados al ser comparados con indicadores del comportamiento financiero de todo sector de la vivienda demuestran tendencias particulares asociadas a dicho comportamiento general.

El documento se ha estructura en seis secciones además de la presente introducción. La segunda sección referencia la metodología utilizada en el presente estudio; la tercera sección realiza una comparación con algunos estudios con un caso particular de la literatura; la cuarta sección presenta una breve descripción del sector para el caso colombiano mediante la explicación de los principales indicadores; la quinta hace una comparación de resultados con el de las fuentes que reportan; y la última sección presenta las diferentes conclusiones. 


\section{Planteamiento metodológico}

De acuerdo con los objetivos planteados para esta investigación se utilizan varios métodos, técnicas estadísticas y uso de instrumentos de medición diferenciados para efectuar el cálculo de los distintos indicadores financieros que sirven de base para realizar los distintos análisis determinando las tendencias y comportamientos de dichos cálculos para las empresas que componen el sector de la construcción.

Varios indicadores contables son establecidos para determinar los distintos comportamientos y tendencias relacionados con la gestión, entre ellos el EVA (Valor Económico Agregado), el vma (Valor del Mercado Agregado), así como indicadores de efectividad, crecimiento, eficacia y eficiencia, que son seleccionados para establecer su incidencia en los distintos resultados para las firmas. La efectividad se mide por los beneficios que origina la inversión: los activos se estudian por el indicador estándar ROA (Rendimiento del Activo) y el patrimonio por el RoE (Rendimiento del Patrimonio) (Ross, et al. 2014; Rivera, 2004). El comportamiento del crecimiento se estable por medio de las utilidades, las ventas y los activos de la empresa.

Los indicadores de eficacia se asocian al comportamiento de las estructuras de gastos y costos, relacionados con las utilidades, como los márgenes bruto, operacional, operacional después de impuestos y neto (Rivera, 2004). Los indicadores de eficiencia determinan la intensidad con la cual las firmas manejan sus activos para originar un adecuado nivel de ventas (Wild, Subramanyam, \& Hasley, 2007). Se usa como principales indicadores la rotación de inventarios, los de cartera, los activos fijos, activos totales y activos operacionales (Rivera, 2004).

El valor económico agregado es un indicador de las utilidades residuales, que resulta de la diferencia entre la utilidad operacional después de impuestos y el cargo por el uso del capital (Stewart, 2000). La relación es:

$$
E V A_{t}=U O D I_{t}-\text { Cargo de capital },
$$

[UODI $\rrbracket_{-\mathrm{t}}$ es la utilidad después de impuestos para un período t; y el componente que resta, es decir, el cargo de capital en el período t se expresa:

$$
\text { Cargo de capital }{ }_{t}=\left(A N O_{t}\right)\left(K o_{t}\right) \text {, }
$$

【ANO $\rrbracket_{-_{t}}$ es el activo neto operacional del período. La variable \ANO $\rrbracket_{-t}$ es la sumatoria del capital de trabajo neto operativo $\llbracket K T N O \rrbracket_{-t}$ y del activo fijo neto operacional $\llbracket A F N O \rrbracket_{-t}$ :

$$
A N O_{t}=K T N O_{t}+A F N O_{t},
$$


De otro lado, [KTNO $\rrbracket_{-}$muestra la diferencia entre los activos corrientes y los pasivos corrientes sin costo explícito. El [AFNO $]_{-}$resulta de restar a los activos fijos operacionales su depreciación. El [ $\left(K_{0}\right]_{-}$es el costo de capital medio ponderado del período t; De acuerdo con Modigliani y Miller (1963), la expresión relevante está determinada por:

$$
K o_{t}=K e(1-L)+K i(1-t) L,
$$

Ke relaciona el costo de oportunidad de acuerdo con la metodología pure play del CAPM (Rivera y Alarcón, 2012). L determina el nivel de endeudamiento de la firma resultante de la relación entre la deuda con costo explícito y el activo neto operacional. $K i$ es el costo de la deuda, este costo debe incluir la deducción impositiva $t$.

De acuerdo con Stewart (2000), el EVA se puede calcular mediante la siguiente expresión:

$$
E V A=\left(A N O_{t}\right)\left[\left(U A I I_{t}\right)(1-t) /\left(A N O_{t}\right)-\left(K o_{t}\right)\right]
$$

$\left([U A I I]_{-}\right)(1-\mathrm{t}) /\left[A N O \rrbracket_{-t}\right.$ es el rendimiento después de impuestos del activo neto operacional. A la diferencia entre el rendimiento después de impuestos del activo neto operacional ANO y el costo de capital medio ponderado [Ko ]_t se le conoce como el margen de utilidad o pérdida residual.

Al trasladar el valor presente EVA de sucesivos periodos, se determina $V M A$ que es el valor de mercado agregado; su expresión se determina de la forma:

$$
V M A=\frac{\sum_{\mathrm{j}=1}^{\mathrm{j}=\mathrm{n}} E V A_{\mathrm{j}}}{(1+\mathrm{Koj})^{\mathrm{j}}}
$$

Se estudia el comportamiento de la gestión del valor con el EVA y sus tres componentes inductores: UODI, ANO y $\kappa_{0}$.

Como complemento, se inspecciona el EVA que crea cada una de las unidades monetarias relacionadas con el $A N O$ con la expresión $E V A / A N O$, al igual que la utilidad operativa después de impuestos requerida para originar determinada unidad monetaria asociada al valor económico agregado originado, con la relación UODI/EVA.

La revisión de la literatura sobre el tema indagado muestra que Rivera y Cuéllar (2018) hallan que el sector de construcción de vivienda en Colombia (SCVC) presenta un incremento de las ventas, activos y utilidad neta, para el período 2010-2016, con una caída de la utilidad neta en el año 2014 (ver tabla 1). 
Tabla 1. Activos, ventas y utilidad neta promedio del scvC

Crecimiento (datos en millones de cop)

\begin{tabular}{|l|ccccccc|}
\hline & 2011 & 2012 & 2013 & 2014 & 2015 & 2016 & Promedio \\
\hline \begin{tabular}{l} 
Ventas \\
\hline $\begin{array}{l}\text { Utilidad } \\
\text { neta }\end{array}$
\end{tabular} & 9.122 & 10.713 & 12.411 & 12.741 & 14.557 & 18.006 & 12.925 \\
\hline Activos & 782 & 969 & 1.215 & 880 & 1.270 & 1.483 & 1.100 \\
\hline
\end{tabular}

Fuente: Rivera y Cuéllar (2018).

Para el periodo 2010-2016 el sector muestra un ROA en términos promedio de 3,3\% y un $R O E$ de 10,1\%, mostrando indicadores satisfactorios para el periodo de referencia. La evolución del ROA y el ROE durante este período lo determinan los márgenes de la utilidad operacional después de impuestos y de la utilidad neta respectivamente. El ROE fue superior al ROA durante todos los años debido al efecto multiplicador del apalancamiento financiero sobre el margen utilidad neta (ver tabla 2).

Tabla 2. Indicadores de efectividad del SCVC

\begin{tabular}{|c|c|c|c|c|c|c|c|}
\hline & 2011 & 2012 & 2013 & 2014 & 2015 & 2016 & Promedio \\
\hline & \multicolumn{7}{|c|}{ a.ROA: Sistema du Pont } \\
\hline \multirow{5}{*}{$\begin{array}{l}R O A(\%) \\
\text { Margen de utilidad operacional } \\
\text { después de impuestos }(\%) \\
\text { Rotación de activos totales (veces) }\end{array}$} & 2,8 & 3,2 & 3,5 & 2,9 & 3,4 & 3,9 & 3,3 \\
\hline & 5,9 & 7,0 & 7,8 & 7,0 & 7,6 & 9,9 & 7,5 \\
\hline & 0,5 & 0,5 & 0,5 & 0,4 & 0,4 & 0,4 & 0,4 \\
\hline & & & & & & & \\
\hline & \multicolumn{7}{|c|}{ b. ROE: Sistema du Pont ampliado } \\
\hline $\operatorname{ROE}(\%)$ & 10,6 & 10,5 & 10,9 & 8,3 & 11,0 & 9,4 & 10,1 \\
\hline Margen de utilidad neta (\%) & 8,6 & 9,0 & 9,8 & 6,9 & 8,7 & 8,2 & 8,5 \\
\hline Rotación de activos totales (veces) & 0,5 & 0,5 & 0,5 & 0,4 & 0,4 & 0,4 & 0,4 \\
\hline Apalancamiento financiero (\%) & 257,0 & 251,8 & 246,2 & 290,0 & 286,5 & 290,1 & 270,3 \\
\hline
\end{tabular}

Fuente: Rivera y Cuéllar (2018, p. 11).

Aunque esta industria tuvo rendimientos positivos, destruyó valor económico en cada uno de los años, debido a que el rendimiento del activo neto operacional fue inferior al costo de capital. El comportamiento del EVA sigue una correlación negativa en relación con la rentabilidad del activo neto operacional (excepto 2016) y con el costo de capital (salvo 2013). Para los años analizados el VMA a comienzo del 2011 reportó un valor de \$-2.974 MM (ver tabla 3). 
Tabla 3. EVA promedio por empresa del SCVC

\begin{tabular}{|lccccccc|} 
& 2011 & 2012 & 2013 & 2014 & 2015 & 2016 & Promedio \\
\hline EVA (millones COP) & -435 & -834 & -859 & -806 & -1.062 & -48 & -674 \\
\hline ANO (millones COP) & 11.374 & 14.213 & 17.225 & 18.493 & 20.337 & 19.818 & 16.910 \\
\hline UODI/ANO (\%) & 4,7 & 5,3 & 5,6 & 4,8 & 5,4 & 9,0 & 5,8 \\
\hline Ko (\%) & 8,5 & 11,1 & 10,6 & 9,2 & 10,7 & 9,3 & 9,9 \\
\hline VMA a 1-1-2011 (millones COP) & -2.974 & & & & & &
\end{tabular}

Fuente: elaboración propia con base en Rivera y Cuéllar (2018), Benchmark, de BPR Asociados Sales \& Credit Management (2017), Superintendencia Financiera (2017) y Damodaran (2017).

\section{Resultados}

En este apartado se presenta la caracterización y la evaluación del desempeño de la pyme del sector construcción de vivienda en Colombia en el período 2011-2016. Este sector se conoce como el de construcción de edificios residenciales (CIIU 4111), que incluye actividades relacionadas con:

- La construcción de todo tipo de edificios residenciales, tales como casas unifamiliares y edificios multifamiliares, incluidos edificios de muchos pisos.

- El montaje y levantamiento in situ de construcciones prefabricadas.

- La reforma o renovación de estructuras residenciales existentes.

- El montaje de cubiertas metálicas, puertas, ventanas y demás elementos metálicos realizado por el constructor como parte del desarrollo de la construcción de edificaciones residenciales. (DANE, 2012, p. 298)

La construcción de edificaciones y de partes de las mismas, así como el acondicionamiento de edificaciones ha crecido en promedio en este periodo cerca de un 6,2\%, por encima del PIB nacional, que fue de 4,2\%. Como se puede apreciar en la figura 1, en cuatro de los seis años analizados ha crecido por encima del promedio de la economía nacional, siendo los años $2013(11,0 \%)$ y 2014 (8,0\%) donde su crecimiento fue mayor. 
Figura 1. Crecimiento del sector construcción de edificios residenciales y del PIB

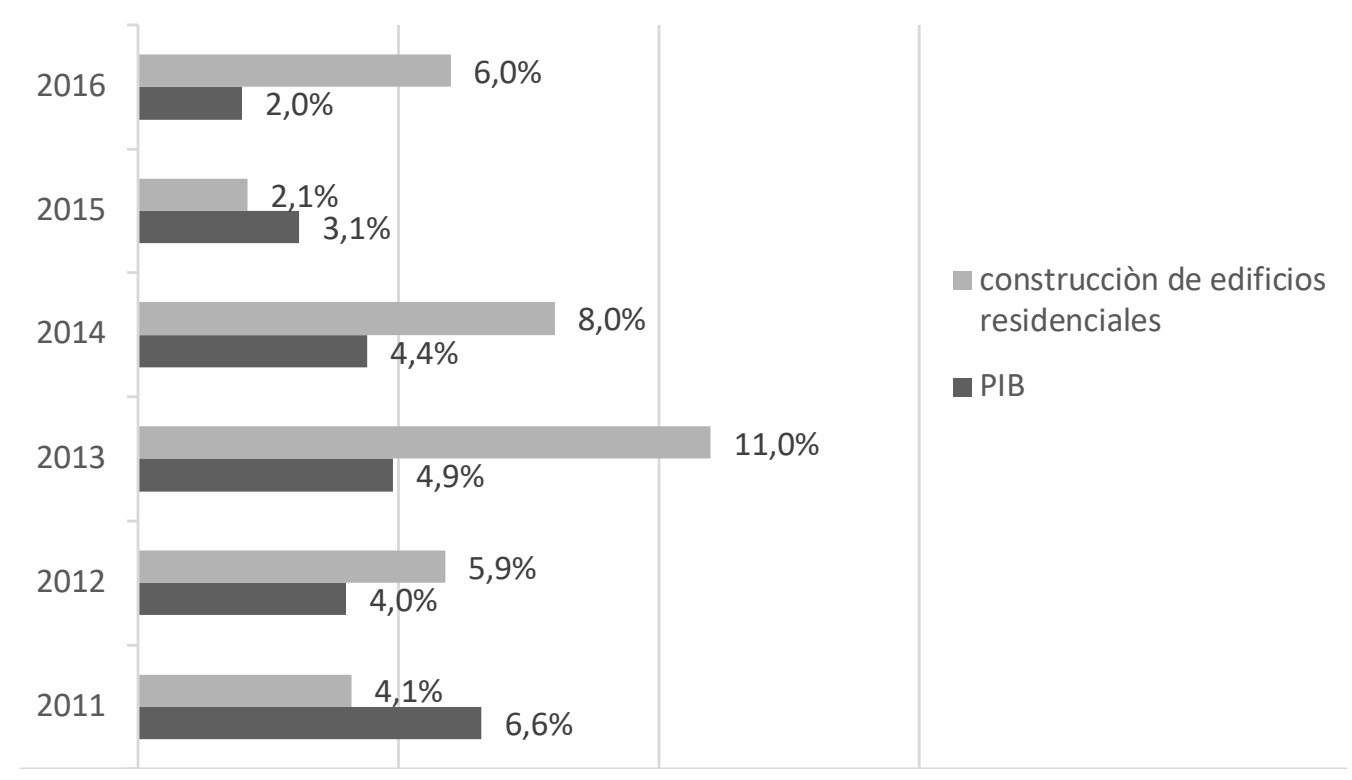

Fuente: elaboración propia con base en DANE (2018).

La participación del subsector construcción de edificios residenciales en relación con el PIB se mantuvo alrededor del 3,1\%, mientras que la contribución de todo el sector construcción (adicionándole la construcción de obras de ingeniería civil) al crecimiento de la economía nacional fue del 6,9\% (ver figura 2).

Tanto la construcción de edificios residenciales como todo el sector de construcción han crecido durante el sexenio. El subsector de construcción de vivienda representó en promedio el 45,3\% del sector construcción en Colombia.

La mayoría de las empresas constructoras en Colombia son pymes. En la tabla 4 se muestra que en el 2014 representaban el $68 \%$ (mediana $43 \%$ y pequeña $25 \%$ ) del total de constructoras, mientras que en el 2016 constituían el 55\% (48\% medianas y 7\% pequeñas). En cuanto a los ingresos operacionales, las pymes registraron el $19 \%$ en el 2014 y $14 \%$ en el 2016. 
Figura 2. Aporte de sector construcción al PIB

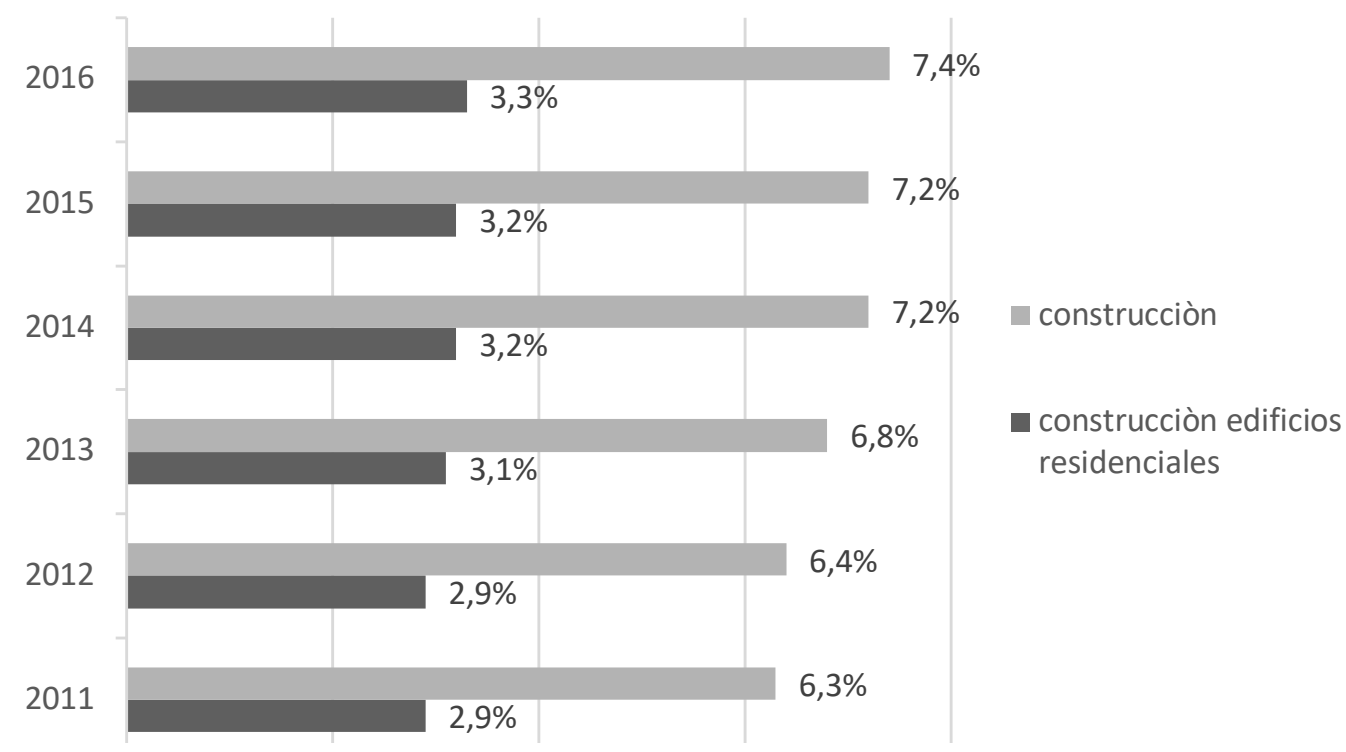

Fuente: elaboración propia con base en DANE (2018).

Tabla 4. Composición del sector construcción por tamaño de empresa año 2014 y 2016

\begin{tabular}{|c|c|c|c|c|}
\hline \multirow[t]{2}{*}{ Tamaño } & \multicolumn{2}{|c|}{ No. empresas } & \multicolumn{2}{|c|}{ Ingresos operacionales } \\
\hline & 2014 & 2016 & 2014 & 2016 \\
\hline Grande & $31 \%$ & $44 \%$ & $81 \%$ & $87 \%$ \\
\hline Mediana & $43 \%$ & $48 \%$ & $16 \%$ & $13 \%$ \\
\hline Pequeña & $25 \%$ & $7 \%$ & $3 \%$ & $1 \%$ \\
\hline Micro & $1 \%$ & $1 \%$ & $0 \%$ & $0 \%$ \\
\hline Total & $100 \%$ & $100 \%$ & $100 \%$ & $100 \%$ \\
\hline
\end{tabular}

Fuente: elaboración propia con base en Superintendencia de Sociedades (2015, p. 8) y Superintendencia de Sociedades (2017, p. 5).

Para determinar las principales relaciones y los cálculos de los indicadores de la pyme del sector construcción en Colombia se toma los estados financieros de un promedio de 186 empresas pequeñas (PCVC) y 441 medianas (MCVC) en el período 2011-2016, reportadas en el anexo de este trabajo. El tamaño se establece conforme al artículo 2 de la Ley 905 de 2004 (Congreso de Colombia, 2004). 


\subsection{Evaluación del crecimiento}

Los activos, ventas y utilidad neta promedio de la pequeña empresa fueron en su orden de 1685 mм\$, 1900 мм\$ y 31 mм\$, mientras que en la mediana empresa estos indicadores fueron correspondientemente de 8882 мM\$, 5827 мм $\$$ y 279 мм\$ (ver tabla 5).

Se observa un aumento continuo de los activos de las pymes en el periodo estudiado, que corresponde a un 54,9\% para la pequeña y a un $37,3 \%$ para la mediana empresa. Las ventas crecieron un $61,2 \%$ en la pequeña y un $31,8 \%$ en la mediana empresa, aunque con caídas entre 2014-2015 en la pequeña, y en el 2016 en la mediana empresa. El crecimiento en activos y ventas es mayor en la pequeña empresa; no obstante, estos valores promedios del periodo son respectivamente cinco y tres veces superiores en la mediana empresa. La utilidad neta de la pyme fue cambiante, mostrando un descenso entre 2015-2016; en los primeros cuatro años esta utilidad fluctúa en la pequeña empresa, pero aumenta en la mediana empresa; lo que demuestra una mayor variabilidad en la pequeña empresa. En términos absolutos del promedio del período se encuentra que la mediana produjo nueve veces la utilidad neta de la pequeña empresa.

Tabla 5. Activos, ventas y utilidad neta promedio

\begin{tabular}{lrrrrrrr} 
& 2011 & 2012 & 2013 & 2014 & 2015 & 2016 & Promedio \\
& \multicolumn{7}{c}{ Crecimiento (millones COP) } \\
Pequeña & & & & & & & \\
Activos & 1.391 & 1.470 & 1.614 & 1.692 & 1.788 & 2.155 & 1.685 \\
Ventas & 1.608 & 1.797 & 1.879 & 1.844 & 1.679 & 2.592 & 1.900 \\
Utilidad neta & 36 & 47 & 32 & 45 & 37 & -8 & 31 \\
Mediana & & & & & & & \\
Activos & 7.306 & 7.986 & 8.681 & 9.605 & 9.677 & 10.035 & 8.882 \\
Ventas & 4.737 & 5.457 & 5.937 & 6.195 & 6.392 & 6.245 & 5.827 \\
Utilidad neta & 217 & 231 & 322 & 327 & 309 & 269 & 279
\end{tabular}

Fuente: elaboración propia. con base en Benchmark, de BPR

Asociados Sales \& Credit Management (2017).

\subsection{Evaluación de la eficiencia}

La pequeña empresa ha rotado su cartera ( 7,6 veces), sus inventarios ( 3,6 veces), sus activos fijos ( 8,0 veces) y sus activos totales ( 1,1 veces) en promedio durante los seis años de estudio, mientras que la rotación promedio en la mediana empresa fue así: cartera (6,2 veces), inventarios ( 1,5 veces), activos fijos (7,5 veces) y activos totales ( 0,7 veces). En promedio, la rotación de los activos en este período ha sido mayor en la pequeña empresa, 
hecho que se replica anualmente con cada uno de categoría de activos, excepto para los activos fijos entre 2013-2015 (ver tabla 6).

La rotación de cada uno de los activos de la pyme oscila con frecuencia en el sexenio, solo la rotación cartera de la pequeña empresa muestra una tendencia a disminuir desde el 2013. No obstante, se encuentra una mayor dispersión en la rotación de la pequeña empresa, con lo que se puede afirmar que la pequeña empresa es más eficiente en el uso de los recursos físicos, aunque con mayores variaciones

\subsection{Evaluación de la eficacia}

Tabla 6. Indicadores de eficiencia

\begin{tabular}{|c|c|c|c|c|c|c|c|}
\hline & 2011 & 2012 & 2013 & 2014 & 2015 & 2016 & Promedio \\
\hline \multicolumn{8}{|c|}{ Rotación (veces) } \\
\hline \multicolumn{8}{|l|}{ Pequeña } \\
\hline $\begin{array}{l}\text { Rotación de cartera } \\
\text { Rotación de inventario } \\
\text { Rotación de activos fijos } \\
\text { Rotación de activos totales }\end{array}$ & $\begin{array}{l}8,1 \\
3,9 \\
7,7 \\
1,2\end{array}$ & $\begin{array}{l}9,6 \\
4,2 \\
7,6 \\
1,2\end{array}$ & $\begin{array}{l}9,1 \\
3,7 \\
6,7 \\
1,2\end{array}$ & $\begin{array}{l}8,0 \\
3,7 \\
7,8 \\
1,1\end{array}$ & $\begin{array}{l}6,8 \\
2,7 \\
7,7 \\
0,9\end{array}$ & $\begin{array}{r}3,7 \\
3,6 \\
10,3 \\
1,2\end{array}$ & $\begin{array}{l}7,6 \\
3,6 \\
8,0 \\
1,1\end{array}$ \\
\hline \multicolumn{8}{|l|}{ Mediana } \\
\hline $\begin{array}{l}\text { Rotación de cartera } \\
\text { Rotación de inventario } \\
\text { Rotación de activos fijos } \\
\text { Rotación de activos totales }\end{array}$ & $\begin{array}{l}6,1 \\
1,4 \\
7,6 \\
0,6\end{array}$ & $\begin{array}{l}6,6 \\
1,7 \\
7,3 \\
0,7\end{array}$ & $\begin{array}{l}6,2 \\
1,6 \\
8,0 \\
0,7\end{array}$ & $\begin{array}{l}7,6 \\
1,4 \\
8,1 \\
0,6\end{array}$ & $\begin{array}{l}7,7 \\
1,4 \\
8,0 \\
0,7\end{array}$ & $\begin{array}{l}3,1 \\
1,4 \\
6,0 \\
0,6\end{array}$ & $\begin{array}{l}6,2 \\
1,5 \\
7,5 \\
0,7\end{array}$ \\
\hline
\end{tabular}

Fuente: elaboración propia con base en Benchmark, de BPR

Asociados Sales \& Credit Management (2017).

En la tabla 7 se puede analizar que los indicadores son positivos en su gran mayoría, excepto en el 2016 para la pequeña empresa. Esto muestra en general que los ingresos de la pyme superan los diferentes costos y gastos. El promedio del sexenio del margen de utilidad bruta en la pequeña empresa $(20,5 \%$ ) fue superior al de la mediana empresas (18,5\%), manteniendo una desviación semejante; mientras que el promedio de los márgenes de utilidad operacional de la pequeña empresa $(4,3 \%)$ fue inferior a de la mediana empresa $(6,6 \%)$, con una desviación más alta en esta última; entre tanto, el margen de utilidad neta en la pequeña empresa $(1,8 \%)$ también estuvo por debajo del logrado por la mediana empresa $(4,8 \%)$, pero con una desviación más alta. 
Tabla 7. Indicadores de eficacia

\begin{tabular}{|c|c|c|c|c|c|c|c|}
\hline & 2011 & 2012 & 2013 & 2014 & 2015 & 2016 & Promedio \\
\hline \multicolumn{8}{|c|}{ Márgenes (\%) } \\
\hline \multicolumn{8}{|l|}{ Pequeña } \\
\hline $\begin{array}{l}\text { Margen de utilidad bruta } \\
\text { Margen de utilidad operacional } \\
\text { Margen de utilidad neta }\end{array}$ & $\begin{array}{r}21,9 \\
4,4 \\
2,2\end{array}$ & $\begin{array}{r}19,5 \\
3,5 \\
2,6\end{array}$ & $\begin{array}{r}20,3 \\
3,7 \\
1,7\end{array}$ & $\begin{array}{r}20,4 \\
5,4 \\
2,4\end{array}$ & $\begin{array}{r}21,1 \\
4,6 \\
2,2\end{array}$ & $\begin{array}{r}20,0 \\
4,1 \\
-0,3\end{array}$ & $\begin{array}{r}20,5 \\
4,3 \\
1,8\end{array}$ \\
\hline \multicolumn{8}{|l|}{ Mediana } \\
\hline $\begin{array}{l}\text { Margen de utilidad bruta } \\
\text { Margen de utilidad operacional } \\
\text { Margen de utilidad neta }\end{array}$ & $\begin{array}{r}17,6 \\
4,8 \\
4,6\end{array}$ & $\begin{array}{r}18,0 \\
5,7 \\
4,2\end{array}$ & $\begin{array}{r}19,8 \\
6,7 \\
5,4\end{array}$ & $\begin{array}{r}19,2 \\
7,4 \\
5,3\end{array}$ & $\begin{array}{r}17,6 \\
7,0 \\
4,8\end{array}$ & $\begin{array}{r}18,7 \\
8,2 \\
4,3\end{array}$ & $\begin{array}{r}18,5 \\
6,6 \\
4,8\end{array}$ \\
\hline
\end{tabular}

Fuente: elaboración propia con base en Benchmark, de BPR

Asociados Sales \& Credit Management (2017).

El porcentaje de gastos de administración y venta fue más alto en la pequeña empresa $(16,5 \%)$ en relación con el de la mediana empresa (11,9\%), teniendo como efecto que la pequeña empresa pasara de tener un margen de utilidad bruta superior, a un margen de utilidad operacional inferior a la de la mediana empresa. El porcentaje de gastos no operacionales de la pequeña empresa $(2,5 \%)$ fue superior a la que se presentó en la mediana empresa $(1,8 \%$ ), lo que condujo a que la inferioridad del margen de utilidad se ampliara más, y presentara un margen de utilidad neta aún más bajo que el mostrado por la mediana empresa.

Los diferentes márgenes de utilidad de la pyme de este sector fluctúan a lo largo del sexenio, y tan solo el margen de la utilidad operacional y el margen de utilidad operacional muestran alguna tendencia: el primero crece, con una caída en el 2015, y el segundo decrece en los tres últimos años.

Estos resultados muestran que la pequeña empresa es más eficaz en el control de los costos de producción y venta, pues saca una diferencia a la mediana empresa de 2,0\% es el margen de utilidad bruta; pero es relativamente menos eficaz en el control de gastos de administración y ventas, porque la mediana pasa aventajarla en 2,3\% en el margen de utilidad operacional; como también es menos eficaz en el control de gastos no operacionales, dado que la mediana empresa supera en un 3,0\% su margen de utilidad neta.

\subsection{Evaluación de la efectividad}

La mediana empresa fue más efectiva en la obtención de rendimientos sobre la inversión, lo anterior se puede contrastar al compararlo con la pequeña empresa: el ROA promedio $(3,0 \%$ vs. $2,0 \%$ ) y el $R O E$ promedio $(9,9 \%$ vs. $3,9 \%$ ) (ver tabla 8$)$. 
Tabla 8. Indicadores de efectividad

\begin{tabular}{|c|c|c|c|c|c|c|c|}
\hline & 2011 & 2012 & 2013 & 2014 & 2015 & 2016 & Promedio \\
\hline \multicolumn{8}{|l|}{ Pequeña } \\
\hline \multicolumn{8}{|c|}{ a. ROA: Sistema du Pont } \\
\hline $\begin{array}{l}R O A(\%) \\
\text { Margen de utilidad operacional } \\
\text { después de impuestos }(\%) \\
\text { Rotación de activos totales (veces) }\end{array}$ & $\begin{array}{l}2,1 \\
1,2\end{array}$ & $\begin{array}{l}1,4 \\
1,2\end{array}$ & $\begin{array}{l}1,6 \\
1,2\end{array}$ & $\begin{array}{l}2,8 \\
1,1\end{array}$ & $\begin{array}{l}1,9 \\
0,9\end{array}$ & $\begin{array}{l}0,8 \\
1,2\end{array}$ & $\begin{array}{l}1,8 \\
1,1\end{array}$ \\
\hline \multicolumn{8}{|c|}{ b. ROE: Sistema du Pont Ampliado } \\
\hline $\begin{array}{l}\text { ROE }(\%) \\
\text { Margen de utilidad neta }(\%) \\
\text { Rotación de activos totales (veces) } \\
\text { Apalancamiento financiero }(\%)\end{array}$ & $\begin{array}{r}6,1 \\
2,2 \\
1,2 \\
237,2\end{array}$ & $\begin{array}{r}6,7 \\
2,6 \\
1,2 \\
210,2\end{array}$ & $\begin{array}{r}4,0 \\
1,7 \\
1,2 \\
206,1\end{array}$ & $\begin{array}{r}5,3 \\
2,4 \\
1,1 \\
202,9\end{array}$ & $\begin{array}{r}4,4 \\
2,2 \\
0,9 \\
211,7\end{array}$ & $\begin{array}{r}-1,0 \\
-0,3 \\
1,2 \\
278,4\end{array}$ & $\begin{array}{r}3,9 \\
1,8 \\
1,1 \\
224,4\end{array}$ \\
\hline \multicolumn{8}{|l|}{ Mediana } \\
\hline \multicolumn{8}{|c|}{ c. ROA: Sistema du Pont } \\
\hline $\begin{array}{l}\text { ROA }(\%) \\
\text { Margen de utilidad operacional } \\
\text { después de impuestos (\%) } \\
\text { Rotación de activos totales (veces) }\end{array}$ & $\begin{array}{l}2,9 \\
0,6\end{array}$ & $\begin{array}{l}3,6 \\
0,7\end{array}$ & $\begin{array}{l}4,3 \\
0,7\end{array}$ & $\begin{array}{l}4,9 \\
0,6\end{array}$ & $\begin{array}{l}4,5 \\
0,7\end{array}$ & $\begin{array}{l}5,5 \\
0,6\end{array}$ & $\begin{array}{l}4,3 \\
0,7\end{array}$ \\
\hline \multicolumn{8}{|c|}{ d. ROE: Sistema du Pont Ampliado } \\
\hline $\begin{array}{l}\text { ROE }(\%) \\
\text { Margen de utilidad neta }(\%) \\
\text { Rotación de activos totales (veces) } \\
\text { Apalancamiento financiero }(\%)\end{array}$ & $\begin{array}{r}9,4 \\
4,6 \\
0,6 \\
317,6\end{array}$ & $\begin{array}{r}8,5 \\
4,2 \\
0,7 \\
294,2\end{array}$ & $\begin{array}{r}10,3 \\
5,4 \\
0,7 \\
278,5\end{array}$ & $\begin{array}{r}11,5 \\
5,3 \\
0,6 \\
336,6\end{array}$ & $\begin{array}{r}10,3 \\
4,8 \\
0,7 \\
324,1\end{array}$ & $\begin{array}{r}9,1 \\
4,3 \\
0,6 \\
338,0\end{array}$ & $\begin{array}{r}9,9 \\
4,8 \\
0,7 \\
314,3\end{array}$ \\
\hline
\end{tabular}

Fuente: elaboración propia con base en Benchmark, de BPR

Asociados Sales \& Credit Management (2017).

El rendimiento del activo de la mediana empresa fue superior a la pequeña empresa en todos los años debido a su mejor eficacia en el manejo de los costos de producción y venta y de los impuestos, lo que le permitió mantener un mayor margen de utilidad operacional después de impuestos $(4,3 \%$ vs. $1,8 \%$ ), y a pesar de su menor eficiencia en el uso de activos (rotación de 0,7 veces vs. 1,1 veces) (ver tabla 8).

$\mathrm{Al}$ analizar el rendimiento del capital propio de la mediana empresa este fue superior a la pequeña empresa en cada año debido a que la mediana empresa fue más eficaz en el control de gastos de administración, venta y otras erogaciones no operacionales, y al uso de un mayor apalancamiento financiero, sin importar que quedara en desventaja respecto a la eficiencia del uso de los activos (ver tabla 8 , parte b y d).

Tanto el ROA como el ROE presentan mayor dispersión en la pequeña empresa, con lo que se puede inferir que la efectividad en el logro de rentabilidades sobre la inversión es más estable en la mediana empresa. 
Revista CIFE / ISSN: 0124-3551 e-ISSN: 2248-4914 / Bogotá-Colombia / Vol. 21 N. o 35 / julio-diciembre 2019 / pp. 141 -165

\subsubsection{EVA de las pequeñas y medianas empresas del sector construcción de vivienda: período 2011-2016}

La pequeña empresa promedio del sector construcción de vivienda destruyó valor económico agregado en todos los años de estudio. Esta destrucción no sigue una tendencia, y surge producto de una UODI cambiante y un cargo de capital en ascenso hasta el penúltimo año. En promedio, se tuvo un Eva anual de -91 millones en el período (ver tabla 9).

Tabla 9. Eva promedio por pequeña empresa y sus inductores

\begin{tabular}{|c|c|c|c|c|c|c|c|}
\hline & 2011 & 2012 & 2013 & 2014 & 2015 & 2016 & Promedio \\
\hline EVA (millones COP) & -40 & -88 & -101 & -83 & -132 & -104 & -91 \\
\hline UODI (millones COP) & 34 & 25 & 31 & 51 & 32 & 20 & 32 \\
\hline Cargo de capital (millones COP) & 75 & 113 & 132 & 134 & 164 & 124 & 124 \\
\hline \multicolumn{8}{|c|}{ a. Inductores del cargo de capital } \\
\hline ANO (millones COP) & 842 & 920 & 1.075 & 1.092 & 1.159 & 1.327 & 1.069 \\
\hline Ko $(\%)$ & 8,9 & 12,2 & 12,3 & 12,3 & 14,2 & 9,3 & 11,5 \\
\hline \multicolumn{8}{|c|}{ b. Indicadores del inductor UODI } \\
\hline UAII (millones COP) & 70 & 62 & 70 & 100 & 77 & 105 & 81 \\
\hline Impuestos (millones COP) & 36 & 38 & 39 & 49 & 44 & 86 & 49 \\
\hline Utilidad bruta (millones COP) & 351 & 350 & 382 & 377 & 355 & 519 & 389 \\
\hline Costo de venta (millones COP) & 1.256 & 1.447 & 1.497 & 1.467 & 1.324 & 2.072 & 1.511 \\
\hline Gastos de admón. (millones COP) & 250 & 256 & 284 & 255 & 250 & 353 & 275 \\
\hline Gastos de venta (millones COP) & 31 & 32 & 27 & 22 & 28 & 46 & 31 \\
\hline \multicolumn{8}{|c|}{ c. Indicadores del inductor ANO } \\
\hline KTNO (millones COP) & 563 & 594 & 681 & 739 & 822 & 1.063 & 744 \\
\hline AFNO (millones COP) & 279 & 325 & 394 & 353 & 337 & 265 & 326 \\
\hline \multicolumn{8}{|c|}{ d. Indicadores del Inductor Ko } \\
\hline $\operatorname{Ke}(\%)$ & 13,5 & 16,5 & 17,1 & 17,0 & 20,4 & 18,1 & 17,1 \\
\hline $\mathrm{Ki}(\%)$ & 7,7 & 9,0 & 7,4 & 7,2 & 8,0 & 11,4 & 8,5 \\
\hline Deuda con costo explícito & & & & & & & \\
\hline (millones COP) & 400 & 340 & 377 & 380 & 429 & 604 & 422 \\
\hline $\mathrm{L}(\%)$ & 47,6 & 37,0 & 35,0 & 34,7 & 37,0 & 45,5 & 39,5 \\
\hline $\mathrm{t}(\%)$ & 50,3 & 44,5 & 55,1 & 52,1 & 54,5 & 110,3 & 61,1 \\
\hline \multicolumn{8}{|c|}{ e. Sistema Dupont del índice UODI/ANO } \\
\hline UODI/ANO (\%) & 4,1 & 2,7 & 2,9 & 4,7 & 2,8 & 1,5 & 3,1 \\
\hline UODI/ventas $(\%)$ & 2,1 & 1,4 & 1,6 & 2,8 & 1,9 & 0,8 & 1,8 \\
\hline Ventas/ANO (veces) & 1,9 & 2,0 & 1,7 & 1,7 & 1,4 & 2,0 & 1,8 \\
\hline \multicolumn{8}{|c|}{ f. Valor del mercado agregado } \\
\hline VMA a 1-1-2011 (millones COP) & -366 & & & & & & \\
\hline
\end{tabular}

Fuente: elaboración propia con base en Benchmark, de BPR Asociados Sales \& Credit Management (2017), Superintendencia Financiera (2017) y Damodaran (2017). 
La tendencia de la UODI está relacionada con el comportamiento de la utilidad operacional para la mayoría de los años menos en el 2016 y por la de los impuestos, menos en el 2012 y 2016 (tabla 10, parte b). La UAII presenta una tendencia similar a la de la utilidad bruta (menos en el año 2014), la utilidad bruta muestra un mismo comportamiento al de las ventas y al del costo de venta a partir del 2013; La UAII sigue un comportamiento inverso a los gastos de venta (excepto en el 2016), y una dirección similar a los gastos de administración en los años 2013, 2015 y 2016 (ver tabla 4 y tabla 9, parte b).

El comportamiento del cargo de capital está alineado con crecimiento continuo del ANO y del Ko, este último solo desciende en el último año, lo que explica la caída del cargo de capital en el 2016 (tabla 9, parte a). El aumento del ANO es afín al comportamiento del KTNO, ya que el AFNO decrece en los últimos tres años. La inversión en AFNO es inferior al KTNO (tabla 9, parte c). La tendencia del Ko es semejante al de las variaciones del Ke en todo el período. La media de Ko fue de 11,5\%, resultante de un Ke de 17,1\%, un Ki del 8,5\%, el endeudamiento es del 39,5\% y una tasa impositiva del 61,1\% (ver tabla 9 , parte d).

$\mathrm{Al}$ examinar el margen de utilidad o pérdida residual se encuentra que el UODI/ANO fue inferior al Ko, para el período, que corrobora por qué la pequeña empresa del sector de construcción de vivienda destruye valor. La fluctuación de UODI/ANO es similar al de la UODI/ventas (ver tabla 9, parte e).

El margen medio de pérdida residual de la pequeña empresa fue de -8,4\%, resultado de la diferencia entre la UODI/ANO promedio (3,1\%) y el Ko promedio (11,5\%).

El vma de la empresa pequeña del sector construcción de vivienda al inicio del 2011 fue de -366 Millones, lo que representa que si bien se generó utilidades netas contables en varios de los años analizados, se destruyó valor (tabla 5 y tabla 9, parte f).

De otro lado la mediana empresa promedio del sector de la construcción de vivienda destruyó EvA de forma cíclica, manteniendo una correlación negativa con el cargo de capital y una correlación positiva con la UODI en el último trienio. El promedio del EVA en el período fue de -275 millones de COP (ver tabla 10, parte b).

La tendencia de la UODI está direccionada por el comportamiento de la utilidad operacional y de los impuestos, excepto con este último en el año 2015. La UAII presenta tendencia equivalente al de la utilidad bruta; esta última sigue una orientación similar a las ventas y costo de ventas hasta el penúltimo año. La UAII fluctúa de manera similar a los gastos de administración (salvo en 2014) y a los gastos de ventas, excepto en el 2016 (ver tabla 10, parte b).

El cargo de capital presenta altibajos, resultado del comportamiento variable del Ko y del aumento del ANO en los primeros cuatro años y su posterior reducción en los dos últimos años (tabla 10, parte a). La dirección del ANO resulta de la combinación del 
aumento del KTNO y el AFNO hasta los años 2015 y 2014 respectivamente, y su posterior disminución hasta final del sexenio. Se observa que el KTNO es superior al AFNO (tabla 10, parte c). Los inductores del Ko muestran que su comportamiento se alinea con la fluctuación del Ki; la Ke también contribuye a la oscilación de Ko, aunque en los años 2014 y 2016 varía en sentido contrario. El promedio de Ko en el período fue de 10,1\%, que resulta de un $\mathrm{Ke}$ de 20,9\%, un $\mathrm{Ki}$ del 8,5\%, un endeudamiento del 70,4\% y una tasa de impuestos del 33,1\% (ver tabla 10, parte d).

$\mathrm{Al}$ evaluar el porcentaje de utilidad residual, se encuentra que UODI/ANO fue menor al Ko en cada uno de los años, esto confirma el motivo por el cual la mediana empresa del sector construcción de vivienda destruye valor en todos los años evaluados. La tendencia de UODI/ANO se explica por el comportamiento de la eficacia en el control de costos y gastos operacionales después de impuestos, dado que mantiene una correlación positiva con el índice UODI/ventas (ver tabla 10, parte e).

Tabla 10. EVA promedio por mediana empresa y sus inductores

\begin{tabular}{|c|c|c|c|c|c|c|c|}
\hline & 2011 & 2012 & 2013 & 2014 & 2015 & 2016 & Promedio \\
\hline EVA (millones COP) & -218 & -300 & -310 & -254 & -324 & -245 & -275 \\
\hline UODI (millones COP) & 139 & 199 & 255 & 302 & 286 & 341 & 254 \\
\hline Cargo de capital (millones COP) & 356 & 498 & 565 & 556 & 610 & 585 & 529 \\
\hline \multicolumn{8}{|c|}{ g. Inductores del cargo de capital } \\
\hline ANO (millones COP) & 4.237 & 4.625 & 5.286 & 5.807 & 5.806 & 5.353 & 5.186 \\
\hline Ko $(\%)$ & 8,4 & 10,8 & 10,7 & 9,6 & 10,5 & 10,9 & 10,1 \\
\hline \multicolumn{8}{|c|}{ h. Indicadores del inductor UODI } \\
\hline UAII (millones COP) & 229 & 311 & 399 & 457 & 447 & 513 & 393 \\
\hline Impuestos (millones COP) & 91 & 113 & 144 & 155 & 161 & 172 & 139 \\
\hline Utilidad bruta (millones COP) & 832 & 984 & 1.175 & 1.189 & 1.123 & 1.168 & 1.079 \\
\hline Costo de venta (millones COP) & 3.904 & 4.473 & 4.762 & 5.006 & 5.270 & 5.077 & 4.749 \\
\hline Gastos de admón. (millones COP) & 532 & 578 & 676 & 616 & 567 & 596 & 594 \\
\hline Gastos de venta (millones COP) & 71 & 95 & 100 & 116 & 109 & 103 & 99 \\
\hline \multicolumn{8}{|c|}{ i. Indicadores del inductor ANO } \\
\hline KTNO (millones COP) & 2.966 & 3.006 & 3.667 & 4.004 & 4.165 & 4.079 & 3.648 \\
\hline AFNO (millones COP) & 1.271 & 1.618 & 1.619 & 1.803 & 1.642 & 1.274 & 1.538 \\
\hline \multicolumn{8}{|c|}{ j. Indicadores del Inductor Ko } \\
\hline $\operatorname{Ke}(\%)$ & 16,0 & 21,3 & 21,2 & 22,8 & 24,4 & 19,9 & 20,9 \\
\hline $\mathrm{Ki}(\%)$ & 7,7 & 9,0 & 7,4 & 7,2 & 8,0 & 11,4 & 8,5 \\
\hline Deuda con costo explícito & & & & & & & \\
\hline (millones COP) & 3.045 & 3.199 & 3.444 & 4.297 & 4.214 & 3.713 & 3.652 \\
\hline $\mathrm{L}(\%)$ & 71,9 & 69,2 & 65,2 & 74,0 & 72,6 & 69,4 & 70,4 \\
\hline $\mathrm{t}(\%)$ & 29,5 & 32,8 & 30,9 & 32,2 & 34,3 & 39,0 & 33,1 \\
\hline \multicolumn{8}{|c|}{ k. Sistema Dupont del índice UODI/AnO } \\
\hline UODI/ANO $(\%)$ & 3,3 & 4,3 & 4,8 & 5,2 & 4,9 & 6,4 & 4,8 \\
\hline UODI/ventas $(\%)$ & 2,9 & 3,6 & 4,3 & 4,9 & 4,5 & 5,5 & 4,3 \\
\hline Ventas/ANO (veces) & 1,1 & 1,2 & 1,1 & 1,1 & 1,1 & 1,2 & 1,1 \\
\hline
\end{tabular}




\begin{tabular}{|c|c|c|c|c|c|c|c|}
\hline & 2011 & 2012 & 2013 & 2014 & 2015 & 2016 & Promedio \\
\hline \multicolumn{8}{|c|}{ f. Valor del mercado agregado } \\
\hline VMA a 1-1-2011 (millones COP) & -1.196 & & & & & & \\
\hline
\end{tabular}

Fuente: elaboración propia con base en Benchmark, de BPR Asociados Sales \& Credit Management (2017), Superintendencia Financiera (2016) y Damodaran (2016).

En términos porcentuales promedios, la pérdida residual fue de $-5,3 \%$ para la mediana empresa, resultado de la diferencia entre la UODI/ANO promedio (4,8 \%) y el Ko prome$\operatorname{dio}(10,1 \%)$.

El valor del mercado agregado del sector construcción de la mediana empresa al inicio del 2011 dio como resultado -1.196 millones de COP, lo que muestra que, si bien se generaron utilidades netas contables en el periodo de referencia, se destruyó valor para cada uno de los años (ver tablas 5 y 10, sección f).

Tanto la PCVC como la MCVC destruyen EvA cada año y en consecuencia generan un valor de mercado agregado negativo para el periodo.

La tabla 11 muestra una serie de indicadores de control para EVA y sus respectivos resultados para determinar las tendencias de la pequeña y la mediana empresa dados los indicadores establecidos.

Tabla 11. Otros indicadores de control del Eva

$\begin{array}{lrrrrrrr} & 2011 & 2012 & 2013 & 2014 & 2015 & 2016 & \text { Promedio } \\ \text { Pequeña } & & & & & & & \\ \text { EVA/ANO } & -0,05 & -0,10 & -0,09 & -0,08 & -0,11 & -0,08 & -0,08 \\ \text { UODI/EVA } & -0,9 & -0,3 & -0,3 & -0,6 & -0,2 & -0,2 & -0,4 \\ \text { Mediana } & & & & & & & \\ \text { EVA/ANO } & -0,05 & -0,06 & -0,06 & -0,04 & -0,06 & -0,05 & -0,05 \\ \text { UODI/EVA } & -0,6 & -0,7 & -0,8 & -1,2 & -0,9 & -1,4 & -0,9\end{array}$

Fuente: elaboración propia con base en Benchmark, de BPR Asociados Sales \& Credit Management (2017), Superintendencia Financiera (2016) y Damodaran (2016).

\section{Discusión}

En esta sección se recopila una serie de elementos de debate de acuerdo con los principales indicadores seleccionados a lo largo del presente estudio. Se recopilan de acuerdo con el crecimiento, la efectividad, el valor económico agregado y sus respectivas relaciones. 


\subsection{Crecimiento}

Los valores promedio de ventas, activos y utilidad neta de la PCVC y la MCVC encontrados en este estudio son inferiores a los que se muestran en el scva, esto debido a que este último no solo considera las pymes, sino también las grandes empresas de este sector en Colombia. La tendencia creciente de las ventas y activos se ha presentado tanto para la SCVC, como para las PGVC y MGVC, donde solo se presentaron caídas puntuales en las ventas de MCVG en el 2016 y de PCVG entre 2014-2015; mientras que el comportamiento de las utilidades netas en los tres grupos fue diferente: creciente en el SCVC (excepto en el último año), creciente (hasta el 2014) y después decreciente en la MGVC, y fluctuante en la PCVC (tabla 12).

Tabla 12. Comparación de los indicadores de crecimiento promedio del sector

\begin{tabular}{lrrrrrrr}
\multicolumn{1}{c}{ Indicador } & 2011 & 2012 & 2013 & 2014 & 2015 & 2016 & Promedio \\
& \multicolumn{7}{c}{ PCVG } \\
Ventas (millones COP) & 1.608 & 1.797 & 1.879 & 1.844 & 1.679 & 2.592 & 1.900 \\
Activos (millones COP) & 1.391 & 1.470 & 1.614 & 1.692 & 1.788 & 2.155 & 1.685 \\
Utilidad neta (millones COP) & 36 & 47 & 32 & 45 & 37 & -8 & 31 \\
& & MCVC & & & & & \\
Ventas (millones COP) & 4.737 & 5.457 & 5.937 & 6.195 & 6.392 & 6.245 & 5.827 \\
Activos (millones COP) & 7.306 & 7.986 & 8.681 & 9.605 & 9.677 & 10.035 & 8.882 \\
Utilidad neta (millones COP) & 217 & 231 & 322 & 327 & 309 & 269 & 279 \\
& & SCVC & & & & & \\
Ventas (millones COP) & 9.122 & 10.713 & 12.411 & 12.741 & 14.557 & 18.006 & 12.925 \\
Activos (millones COP) & 19.037 & 23.189 & 27.509 & 30.644 & 32.933 & 45.897 & 29.868 \\
Utilidad neta (millones COP) & 782 & 969 & 1.215 & 880 & 1.270 & 1.483 & 1.100
\end{tabular}

Fuente: Ver tablas 1 y 5.

\subsection{Efectividad}

$\mathrm{Al}$ confrontar el ROA medio de la SCVG $(3,3 \%)$, la PGVC $(2,0 \%)$ y la MCVC $(3,0 \%)$, se aprecia que todo el sector es más efectivo que la pyme. Esto se debe a que es más eficaz en el control de las erogaciones operacionales, que le permite compensar su menor eficiencia en el uso de los activos. La mediana empresa es más efectiva que la pequeña empresa porque es más eficaz en la gestión de costos y gastos operacionales, a pesar de ser menos eficiente en la utilización de los activos que la pequeña empresa (ver tablas 13, 8 y 2). 
Tabla 13. Comparación de los indicadores de efectividad de la industria

\begin{tabular}{|c|c|c|c|c|c|c|c|}
\hline Indicador & 2011 & 2012 & 2013 & 2014 & 2015 & 2016 & Promedio \\
\hline \multicolumn{8}{|c|}{ PCVC } \\
\hline $\operatorname{ROA}(\%)$ & 2,5 & 1,7 & 1,9 & 3,0 & 1,8 & 0,9 & 2,0 \\
\hline ROE $(\%)$ & 6,1 & 6,7 & 4,0 & 5,3 & 4,4 & $-1,0$ & 3,9 \\
\hline \multicolumn{8}{|c|}{ MCVC } \\
\hline $\mathrm{ROA}(\%)$ & 1,9 & 2,5 & 2,9 & 3,1 & 3,0 & 3,4 & 3,0 \\
\hline $\operatorname{ROE}(\%)$ & 9,4 & 8,5 & 10,3 & 11,5 & 10,3 & 9,1 & 9,9 \\
\hline \multicolumn{8}{|c|}{ SCVC } \\
\hline ROA $(\%)$ & 2,8 & 3,2 & 3,5 & 2,9 & 3,4 & 3,9 & 3,3 \\
\hline ROE $(\%)$ & 10,6 & 10,5 & 10,9 & 8,3 & 11,0 & 9,4 & 10,1 \\
\hline
\end{tabular}

Fuente: ver tablas 2 y 8.

De otro lado, al examinar el RoE medio del scva (10,1\%), la PCVG (3,9\%) y la MCVG $(9,9 \%)$, se encuentra que el scva es el más efectivo para sus propietarios, debido a que logra ser más eficaz en el control de todos los costos y gastos que la pyme, además saca ventaja sobre la PCVC al hacer uso de un mayor apalancamiento financiero positivo. La MGVC es más efectiva para sus dueños que la PGVC, pues logra una mayor eficacia en la gestión de todas las erogaciones, además de disponer del mayor apalancamiento financiero (incluso por encima del presentado por el SCVC). A pesar que la PCVC fue la más eficiente en el uso de los activos, seguida de la MCVG, este factor fue el que menos tuvo peso para determinar qué tan efectivo es cada uno de estos grupos para sus propietarios (ver tablas 13,8 y 2 ).

\subsection{Valor económico agregado}

El Eva del scve es negativo en todos los años evaluados, lo mismo sucede con las pymes. La destrucción del Eva es fluctuante manteniéndose una correlación positiva entre el SCVG, la PGVG y la MGVG (ver tabla 14).

Examinando los inductores del EVA se halla que la relación UODI/ANO de la PCVC no sigue una orientación determinada; mientras que en la MCVC y en el SCVC crecen con caídas en un solo año: en el 2015 en la primera y en el 2014 en el segundo.

El Ko de los tres grupos es cambiante, aunque solo entre el SCVC y la MCVC las variaciones son semejantes en los primeros cinco años (ver tabla 14).

Dados los grupos de referencia para los tres grupos se encuentra que el scva presenta la mayor rentabilidad de activo operacional (5,8\% y el menor costo de capital 9,9\%). De 
Revista CIFE / ISSN: 0124-3551 e-ISSN: 2248-4914 / Bogotá-Colombia / Vol. 21 N.o 35 / julio-diciembre 2019 / pp. $141-165$

otro lado, la PCVC muestra la menor rentabilidad del ANO (3,1\%), y el mayor costo de capital $11,5 \%$; lo que da como resultado que el porcentaje de pérdida residual media sea más alta en la PCVC (-8,4\%), seguida de la MCVC de $-5,3 \%$ y de la calculada por el scVG de $-4,1 \%$.

Tabla 14. Comparación del EVA promedio por empresa del sector y sus inductores

\begin{tabular}{|c|c|c|c|c|c|c|c|}
\hline Indicador & 2011 & 2012 & 2013 & 2014 & 2015 & 2016 & Promedio \\
\hline \multicolumn{8}{|c|}{ PCVG } \\
\hline EVA (millones COP) & -40 & -88 & -101 & -83 & -132 & -104 & -91 \\
\hline UODI/ANO $(\%)$ & 4,1 & 2,7 & 2,9 & 4,7 & 2,8 & 1,5 & 3,1 \\
\hline Ko $(\%)$ & 8,9 & 12,2 & 12,3 & 12,3 & 14,2 & 9,3 & 11,5 \\
\hline ANO (Millones COP) & 842 & 920 & 1.075 & 1.092 & 1.159 & 1.327 & 1.069 \\
\hline \multicolumn{8}{|c|}{ MCVC } \\
\hline EVA (millones COP) & -218 & -300 & -310 & -254 & -324 & -245 & -275 \\
\hline UODI/ANO (\%) & 3,3 & 4,3 & 4,8 & 5,2 & 4,9 & 6,4 & 4,8 \\
\hline $\mathrm{Ko}(\%)$ & 8,4 & 10,8 & 10,7 & 9,6 & 10,5 & 10,9 & 10,1 \\
\hline ANO (Millones COP) & 4.237 & 4.625 & 5.286 & 5.807 & 5.806 & 5.353 & 5.186 \\
\hline \multicolumn{8}{|c|}{ SCVG } \\
\hline EVA (millones COP) & -435 & -834 & -859 & -806 & -1.062 & -48 & -674 \\
\hline UODI/ANO $(\%)$ & 4,7 & 5,3 & 5,6 & 4,8 & 5,4 & 9,0 & 5,8 \\
\hline Ko $(\%)$ & 8,5 & 11,1 & 10,6 & 9,2 & 10,7 & 9,3 & 9,9 \\
\hline ANO (Millones COP) & 11.374 & 14.213 & 17.225 & 18.493 & 20.337 & 19.818 & 16.910 \\
\hline
\end{tabular}

Fuente: elaboración propia e información de las tablas 3, 9, 10.

\section{Conclusiones}

Para el análisis realizado a las pymes del sector construcción de vivienda en Colombia en el período 2011-2016 se encontró que los activos para las medianas y las pequeñas firmas tienden a crecer; las ventas y utilidades netas de la pequeña empresa fluctúan, mientras que en la mediana empresa crecen, pero caen al finalizar el sexenio.

Las pymes asociadas a la construcción de vivienda mostraron indicadores contables favorables para el periodo estudiado al demostrar, en términos de promedios, rendimientos y utilidades positivos. No obstante, en términos generales MGVC muestra una mayor relación de efectividad que la PGVC en la obtención de utilidades de acuerdo con la inversión, usando cualquiera de las medidas tradicionales como el ROA o el ROE. Esto se debe a la ventaja que saca la MCVG sobre la PGVC en los siguientes factores, citados en orden de relevancia: 1) eficacia en la gestión de todos los costos y gastos (operacionales y no operacionales); 2) mantener siempre un apalancamiento financiero más alto y positivo.

Sin embargo, medidas relacionadas con la efectividad de la pyme es más alta en los cálculos de SCVC si se valora con cualquiera de los indicadores asociados a la ROA o el ROE. Esto se debe a que el scva presenta una mayor eficacia en la gestión de todos los costos 
y gastos en comparación a la PCVC y a la MCVC; además de contar con mayor grado de apalancamiento financiero que la PGVG.

Los cálculos muestran un buen desempeño financiero de la pyme de este sector dadas las estimaciones de los indicadores contables. Sin embargo, algunos de los resultados desestiman el buen comportamiento general dada la pérdida que se registra en algunos de los indicadores asociados con el valor económico agregado tanto para la mediana como para la pequeña empresa; la explicación se puede encontrar en los resultados de las pérdidas residuales para el periodo de estudio.

Caso equivalente acontece con el scva que muestra indicadores contables propicios, en utilidades, ROA y ROE; sin embargo, los resultados para el EVA no son satisfactorios, para los propietarios, que en el sexenio han tenido un vMA negativo. Esto es consecuencia de presentar un indicador UODI/ANO menor al Ko cada año.

En términos absolutos, la MGVC destruye mayor Eva que la PGVC, pero cuando se relaciona con el ANO es al contrario; la PCVC destruye mayor más valor por peso invertido.

\section{Referencias bibliográficas}

BPR Asociados Sales, \& Credit Management (2017). Benchmark. [base de datos en línea]. Recuperado el 20 de noviembre de 2017 de http://bck.securities.com/ mainview? sector_id=9999028\&sv $=$ BCK\&pc $=\mathrm{CO}$

Congreso de Colombia (agosto de 2004). Ley 905 de 2004.

Damodaran, A. (2017). [Base de datos en línea]. Recuperado el 14 de diciembre de 2017 de http:/ /pages.stern.nyu.edu/ adamodar/

DNP (2017). Plan nacional de desarrollo 2010-2014. Prosperidad para todos. Resumen ejecutivo. https://colaboracion.dnp.gov.co/CDT/PND/Resumen \%20Ejecutivo\%20 Ultima\%20Version.pdf

DANE (2012). Clasificación industrial internacional uniforme de todas las actividades económicas. Revisión 4 adaptada para Colombia. CIIU Rev. 4 A.C. Recuperado el 15 de diciembre 2016 de https://www.dane.gov.co/files/nomenclaturas/CIIU_Rev4ac.pdf

DANE (2018). Boletín técnico IV trimestre del año 2017. Recuperado el 20 de junio de 2018 de https://www.dane.gov.co/index.php/estadisticas-por-tema/cuentas-nacionales/ cuentas-nacionales-trimestrales/historicos-producto-interno-bruto-pib\#base-2005 


\section{"Čl'F́'E 35}

Revista CIFE / ISSN: 0124-3551 e-ISSN: 2248-4914 / Bogotá-Colombia / Vol. 21 N. o 35 / julio-diciembre 2019 / pp. 141 -165

Martin, J., \& Petty, J.,(2001): La gestión basada en el valor. La respuesta de la empresa a la revolución del accionista. Barcelona: Gestión 2000.

Milla, A. (2010). Creación de valor para el accionista. España: Ediciones Díaz de Santos.

Modigliani, F., \& Miller, M. (1963). Corporate income taxes and the cost of capital: a correction. The American Economic Review, 53, 433-443.

Revista Semana (2016, octubre 12). La vivienda, el motor del país. Recuperado el 20 de junio de 2017 de https://www.semana.com/economia/articulo/politica-de-vivienda-la-locomotora-mas-importante-de-la-economia-nacional/509339

Rivera, J. (2004). Introducción a la administración financiera: fundamentos y aplicaciones para crear valor. Cali: Universidad del Valle.

Rivera, J., \& Alarcón, D. (2012). El cargo de capital en la evaluación del desempeño financiero de empresas innovadoras de confecciones de Cali. Estudios Gerenciales, 38(123), 85-100.

Rivera, J., \& Cuéllar, D. (2018). Sector construcción de edificios residenciales en Colombia: comportamiento financiero durante el período 2011-2016. Documento no publicado.

Ross, S., Westerfield, R., \& Jordan, B. (2014). Fundamentos de finanzas corporativas (10. ${ }^{\text {e edi- }}$ ción). México: McGraw-Hill Interamericana.

Stewart, B. (2000). En busca del valor. Barcelona: Ediciones Gestión 2000.

Superintendencia de Sociedades. (2015). Desempeño del sector de construcción edificaciones 2012 - 2014. Informe. Recuperado el 20 de noviembre de 2017 de https:// www.supersociedades.gov.co/noticias/Documents/2015/EE4-\%20Estudio\%20 construcci $\%$ C3\% $\% 33$ n $\% 20$ edificaciones- $\% 202015 \% 20 \mathrm{VII} \% 2010$.pdf

Superintendencia de Sociedades. (2017). Delegatura de Asuntos Económicos y Contables. Grupo de Estudios Económicos y Financieros. Desempeño del sector construcción edificaciones informe. Recuperado el 20 de noviembre de 2017 de https://incp.org. co/Site/publicaciones/info/archivos/construccion-de-edificaciones.pdf

Superintendencia Financiera de Colombia. (2017). Tasa de interés y desembolsos por modalidad de crédito. Recuperado el 18 de diciembre de https://www.superfinanciera.gov. $\mathrm{co} /$ jsp $/$ loader.jsf ? lServicio $=$ Publicaciones\&lTipo $=$ publicaciones\&lFuncion $=$ load $\mathrm{C}$ ontenidoPublicacion\&id=60955

Wild, J., Subramanyam, K., \& Hasley, R. (2007). Análisis de estados financieros (9a. edición). México: McGraw-Hill Interamericana. 


\section{Anexo. Pyme del sector construcción de vivienda en Colombia}

\begin{tabular}{|c|c|c|c|c|c|c|c|c|}
\hline 830.063.227; & 900.514 .667 & 860.509 .527 & 900.122.283; & 900.329 .527 & 900.386 .571 & 860.063.108; & 830.000.129; & 813.003.433; \\
\hline 900.536 .058 & 900.536.166; & 805.029.393; & 890.212.254; & 890.914.965; & 900.448.438; & 900.186 .150 & 860.036.730; & 830.147.492; \\
\hline 04.003.640; & 805.010.369; & 900.439 .501 & 805.014.656; & 900.355 .526 & 860.402 .922 & 800.022 .115 & 900.084 .460 & 0.000 .135 \\
\hline 10.006.108; & 900.100.443; & 800.093.266; & 800.237.294; & 830.508.765; & 800.078.011; & 900.220 .598 & 800.113.213; & 900.444 .014 \\
\hline 30.043.648; & 800.006.608; & 890.904.041; & 805.029.905; & 860.535.490; & 900.270 .826 & 900.521 .357 & 900.179.804; & 900.617 .372 \\
\hline 11.025.261; & 830.130.119; & 830.064.222; & 900.221.267; & 811.045.749; & 800.196.811; & 800.177.215; & 900.062 .453 & 0.098 .143 \\
\hline 00.265 .023 ; & 890.506.038; & 900.028 .123 & 900.087 .656 & 800.208.210; & 900.375.704; & 891.855.774; & 802.013.829; & 900.498.388; \\
\hline 00.030.829; & 900.141.829; & 900.140 .015 & 900.578.885; & 900.172 .213 & 891.101.262; & 900.173.385; & 900.480 .366 & 900.297.952; \\
\hline 00.070 .124 & 830.060.858; & 805.031.260; & 800.045.458; & 900.301 .745 & 830.017.927; & 900.138 .413 & 900.035 .722 & 830.509.518; \\
\hline 17.002.218; & 800.192.961; & 830.015.815; & 890.003.343; & 900.259.154; & 900.125 .860 & 830.010.707; & 900.396.901; & .387 \\
\hline 00.433 .648 & 811.026.526; & 900.022 .943 & 900.144.205; & 811.026.321; & 900.070 .710 & 890.211.614; & 900.138 .748 & 900.609.064; \\
\hline 04.001.380; & 900.547 .686 & 800.188.665; & 900.343.072; & 830.142.662; & 860.045 .574 & 830.096.389; & 3.186 & 174 \\
\hline 30.067.699; & 830.133.698; & 890.909.034; & 800.149.556; & 900.116 .592 & & 900.098.684; & 46.118; & 4.661; \\
\hline 00.311.388; & 800.053.720; & 800.141.409; & 900.130.997; & 900.447 .557 & 800.010.983; & 900.208 .746 & 800.128.984; & 900.445 .624 \\
\hline 00.190 .263 & 900.100 .748 & 900.084 .629 & 800.018.411; & 830.123.946; & 900.228 .666 & 900.291.484; & 830.032.573; & 860.005 .462 \\
\hline 4.496 & 900.164.492; & 900.103.879; & 900.457.817; & 900.554 .451 & 860.049.837; & 800.165.388; & 2.246 & 843; \\
\hline .249 & 900.290.906; & 1.325; & 816.007.125; & & .060 & 587 & 8.085; & .369 ; \\
\hline 00.200.571; & 800.030.893; & 900.060 .879 & 900.227.321; & 800.089.522; & 830.504.751; & 900.430 .960 & 900.073 .266 & $.951 ;$ \\
\hline 353 & 804.001.145; & 900.227 .331 & 890.939.454; & 860.058.303; & & 900.572 .013 & & .579 \\
\hline .584 & 900 & 805 & 830.1 & 800. & & & & \\
\hline 00.559 .547 & 800.034.638; & 900.5 & & & 0.012 .771 ; & & & 14 \\
\hline 00.049.254; & 900.412.260; & 830.125.005; & 900.198.914; & 60.204 & 0.347 .424 & 830 & & 453; \\
\hline 673 & 802.020.619; & 900.469.266; & 900.472.288; & 860. & & 900 & & 234; \\
\hline 457; & 900. & 900. & 900. & & & 09; & & \\
\hline 00.132.687; & 900.399.929; & 900.568 .627 & 860.350.883; & 860. & & & 185; & .752 \\
\hline 00.051 .984 & 900.444.106; & 800.196.687; & 800.112.784; & 12 & .707 & 918; & 978; & $691 ;$ \\
\hline 750 & 800.252.523; & 805.0 & 892.3 & 900 & 286 & & & 69 \\
\hline & & & & & & & & \\
\hline .584 & 900.023.788; & 900.663 .554 & & 388 & & 69 & & 338; \\
\hline .652 & 890.101.131; & 900.471.213; & 890.1 & 900. & 827; & 781; & 29; & $602 ;$ \\
\hline $.113 ;$ & 900.181 .580 & 900.076 .995 & 900.088.623; & 2.232; & .744 & 080 & & 648 \\
\hline & & & & & & & & \\
\hline .096; & 800.112.084; & 900.137 .583 & 900. & 51 & & 80 & & 622; \\
\hline 300.183.064; & 900.362.118; & 892.200.725; & 890.3 & 527 & $122 ;$ & 41 & & 659; \\
\hline 300.183.970; & 806.008.712; & 900.221 .138 & 900.634.624; & 890.942.101; & 007.202 & 27 & & 442; \\
\hline & & & & & & & & \\
\hline .322 & 830.119.070; & 800.090.652; & 900.540 .745 & 37.803; & & 50 & 288; & 71; \\
\hline .049 & 900.465.296; & 860.400 .572 & 900.517.529; & 900.5 & 38 & 56 & & 304; \\
\hline 900.339.535; & 900.480.908; & 830.065.587; & 860.353.170; & 900.054 .240 & 900.258.343; & 214 & & 980 ; \\
\hline & & & & & & & & \\
\hline 900.537 .884 & 900.074.158; & 900.321 .110 & 900.619.657; & 860.069.067; & 32 & 3.786; & 385; & 306; \\
\hline 300.181.181; & 900.149.425; & 807.004.759; & 830.006.224; & 900.450 .600 & 900.169.529; & 1.205; & 90 & 008 ; \\
\hline 00.133 .059 & 900.327.596; & 800.250 .176 & 900.434.793; & 891.408.425; & 900.480 .356 & 005.652 & 90 & 431; \\
\hline & 800. & & & & & & & \\
\hline 00.156 .253 & 830.039.109; & 800.243.902; & 900.299.782 & 900.339.082; & 900.352 .525 & 19; & 88 ; & 330; \\
\hline 00.318 .759 & 811.011.704; & 900.435.003; & 900.128.496; & 900.601 .152 & 800.152.650; & 74 & 11; & 996 ; \\
\hline 00.468.590; & 900.367.085; & 900.310 .533 & 811.038.832; & 860.030.872; & 900.164.977; & 001.795 & 90 & 80 \\
\hline & 900. & & 80 & & & & & \\
\hline 900.470 .628 & 900.588.674; & 900.223 .683 & 830.092.955; & 900.100 .967 & 800.019.572; & 900.038 . & 90 & 206; \\
\hline 360.516.088; & 900.120.978; & 900.101 .354 & 900.270.696; & 830.002.490; & 860.050.591; & 860.505.864; & 822.002.440; & .410 \\
\hline 900.219 .992 & 890.940.910; & 860.003.709; & 811.024.630; & 900.424 .218 & 900.376 .834 & 900.153.088; & 73.411; & 511; \\
\hline & 890. & & 89 & & & & & \\
\hline 00.153 .599 & 890.213.122; & 900.575 .130 & 900.015 .682 & 860.070 .320 & 900.699 .522 & 900.433 .100 & 54 & 445; \\
\hline 00.600.608; & 830.119.339; & 890.204.988; & 822.005.667; & 900.108 .123 & 900.119 .316 & 900.377 .378 & 830 & 800.1 \\
\hline & 860.041.391; & 800.058.474; & 800.058.369; & 900.110 .934 & 900.378.289; & 900.416.440; & 860 & 900. \\
\hline & 900.630.475; & 900. & & & & & & \\
\hline 800.047.781; & 802.003.326; & 900.423 .764 & 900.459.060; & 900.402.178; & 900.178.588; & 900.216 .962 & 900.209.747; & 860. \\
\hline 900.482.807; & 805.031.242; & 830.089.099; & 800.041.356; & 900.191.597; & 900.509.198; & 900.527 .261 & 900.397.224; & 900.230 .779 \\
\hline & 830.511.960; & 817.000.947; & 900.577 .476 & 800.195.032; & 800.051.659; & 900.115.895; & 900. & 800 \\
\hline & 900.1 & 830.136 & & & & & & \\
\hline & 811.022.841; & 900.551 .953 & 813.002.772; & 900.305.329; & 900.228.458; & 890.203.463; & 900.592.386; & 830.143.509; \\
\hline 900.345 .117 & 804.017.067; & 900.296.839; & 801.002.376; & 900.624 .997 & 900.553 .647 & 811.001.017; & 830.070.959; & 804.015.099; \\
\hline 37.689 & 900.588.604; & 900.256 .204 & 900.497.620; & 900.073 .719 & 900.427.085; & 800.053.915; & 900.485.511; & 830.104.051; \\
\hline & 890.503.178; & 890.303 .7 & 891.411.023; & 900.411 .468 & 900.415.979; & 900.137.167; & 900 & 800.2 \\
\hline & 800.188.715; & 830.032.610; & 900.170.742; & 800.064 .177 & 800.136.956; & 860.510.914; & 900.604.146; & 900.188.165; \\
\hline 800.057.165; & 900.278.934; & 900.364 .362 & 890.929.487; & 900.389.088; & 900.403.824; & 900.153.161; & 809.011.598; & 900.237 .104 \\
\hline 900.283 .603 & 801.001.608; & 900.368 .657 & 800.228.069; & 830.116.891; & 900.201 .727 & 900.458 .625 & 900.409.239; & 891.501.427; \\
\hline & 822.001.280; & 890.406.657; & 890.923.681; & 900.600 .284 & 830.090.978; & 900.278 .611 & 900.61 & 900.480 .140 \\
\hline 900.256.940; & 900.574 .218 & 800.116.050; & 809.009.469; & 900.410 .434 & 890.506.325; & 900.595 .651 & 900.223.883; & 900.482.179; \\
\hline 900.193.989; & 830.020.495; & 819.003.158; & 900.236.687; & 900.508 .366 & 891.501.783; & 900.577 .887 & 900.061.323; & 830.068 .06 \\
\hline 890.936.738; & 804.002.491; & 900.502 .215 & 800.071.745; & 900.236.755; & 892.200.328; & 890.937 .515 & 900.312 .9 & 811.014.812; \\
\hline
\end{tabular}


Revista CIFE / ISSN: 0124-3551 e-ISSN: 2248-4914 / Bogotá-Colombia / Vol. 21 N.o 35 / julio-diciembre 2019 / pp. 141 -165

\begin{tabular}{|c|c|c|c|c|c|c|c|c|}
\hline 900.555 .836 & 830.002.608; & 800.158.791; & 900.112 .154 & 900.683 .456 & 900.563 .955 & 802.005.438; & 860.077.215; & 830.066.664; \\
\hline 900.052 .107 & 900.302 .097 & 900.528 .823 & 890.117.198; & 800.191.462; & 900.160 .972 & 830.124.332; & 900.382 .623 & 802.007.729 \\
\hline 900.427 .660 & 900.303 .461 & 811.033.699; & 860.525.678; & 860.091.073; & 900.459.480; & 830.121.265; & 900.341.530; & 900.595 .817 \\
\hline 860.025.054; & 900.152 .293 & 900.488 .742 & 810.004.017; & 900.674 .442 & 900.103 .513 & 900.385 .456 & 830.020.375; & 860.014.156 \\
\hline 802.020.239; & 801.004.232; & 800.251.267; & 890.116.722; & 830.009.905; & 830.143.733; & 900.465.804; & 900.434 .646 & 900.129 .676 \\
\hline 800.230.511; & 800.185.685; & 900.188 .351 & 830.026.194; & 900.184 .622 & 830.505.170; & 800.201.623; & 900.279 .148 & 860.354 .536 \\
\hline 800.239.598; & 812.000.120; & 830.125.085; & 800.085.734; & 830.142.715; & 900.394.886; & 802.020.651; & 900.415 .490 & 813.002.489 \\
\hline 900.023 .366 ; & 890.104.625; & 900.511 .465 & 900.390.773; & 830.512 .580 & 900.508.904; & 810.006.760; & 900.430.808; & 800.088.209; \\
\hline 800.1 & 900.352 .754 & 816.000.920; & 804.004.477; & 900.398.569; & 860.350.617; & 800.129.643; & 800.087.240; & 900.017 .348 \\
\hline 830.0 & 805.028.121; & 900.527 .319 & 811.033.146; & 900.278 .754 & 900.194 .001 & 900.457.350; & 900.051 .754 & 830.117.411; \\
\hline 830.1 & 860.045.744; & 830.136.915; & 800.231.486; & 900.006 .157 & 900.159.710; & 800.253.099; & 830.506.825; & 890.804.399; \\
\hline 890. & 900.526 .112 & 900.438 .524 & 900.243 .718 & 811.031.963; & 900.127.494; & 900.143.889; & 900.280 .465 & 830.072.373; \\
\hline 830.120.025; & 900.431.973; & 832.010.507; & 830.141.348; & 800.156.976; & 830.108.980; & 900.115.859; & 802.018.269; & 891.855.782 \\
\hline 830.507.325; & 900.411.991; & 805.024.057; & 830.504.077; & 830.009.027; & 900.070 .102 & 900.264 .647 & 804.004.908; & 830.084.501; \\
\hline 900.0 & 830.117.707; & 900.444 .877 & 890.270.773; & 890.704.501; & 860.514.361; & 900.056 .050 & 900.171 .539 & 800.230 .127 \\
\hline 900. & 900.526.834; & 800.138.870; & 800.229.201; & 802.020.447; & 900.008.999; & 900.201.933; & 3.744; & 844.001.418; \\
\hline 830.0 & 900.211.123; & 900.186 .240 & 890.207.801; & 14.142; & 900.637 .056 & 0.532 & .349 & 30.232 \\
\hline 860.528.092; & 900.314.735; & 900.554.899; & 800.219.778; & 900.538 .755 & 830.055.175; & 891.101.301; & 830.085.282; & 800.234 .857 \\
\hline 805.015.121; & 900.078 .768 & 900.596 .925 & 830.014.921; & 830.070.777; & 860.048.944; & 816.006.026; & 800.195.337; & 860.354 .612 \\
\hline 900. & 900.349 .820 & 805.025.557; & 830.035.267; & 830.106.627; & 3.175; & .568 & 2.214 & 437 \\
\hline 900 & 800 & 900. & 900. & 811.038.269; & 820.000.187; & 830.503.526; & 3.376 & 1.480; \\
\hline 900. & 900.267.414; & 900.065.878; & 804.006.294; & 900.317 .686 & 860.354.467; & 813.013.767; & 830.052.489; & 860.508.146; \\
\hline 801. & 860.016.593; & 900.441 .668 & 900.516 .149 & 900.384 .314 & 900.719 .828 & 900.464.813; & 900.257.081; & 900.031 .232 \\
\hline 900. & 830.063.017; & 891.409.028; & 830.144.453; & 892.301.810; & .953 & & 8.651; & .593 \\
\hline 890 & 14; & & 860 & & & & & 149 \\
\hline 800.1 & 900.155.175; & 830.002.362; & 890.921.300; & & & & & \\
\hline 800.0 & 900.040 .184 & 800.179.292; & 800.090.716; & 800.163.387; & 900.239.134; & 830.508.088; & 3.258; & 802 \\
\hline 830.0 & 900.515 .074 & 830.117.896; & 800.119.054; & 890.1 & 90 & 900 & 900 & 830 \\
\hline 822. & & 3.444; & & & & & 59 ; & \\
\hline 900 & 860 & 900. & 800. & 830. & 800.008.400; & 900. & 3.573; & 041 \\
\hline 811. & 900.480 .471 & 900.280 .222 & 800.124.975; & 800.141.509; & 900.035.085; & 4.409; & 9.014; & .472 \\
\hline 830.0 & 890.000.556; & 800.174.951; & 830.032.668; & 800.254.154; & 800.014.903; & 900.329 .161 & 9.242 & 800.107.604 \\
\hline 860.0 & & 830.018.803; & 830.145.983; & 900.120 .363 & 187; & 4.601; & 35 & \\
\hline 900. & 86 & 806 & 800. & 890 & 218 & 830 & 491; & 50 \\
\hline 900. & 900.360 .342 & 802.022.773; & 830.114.388; & 900.131.238; & 800.158.609; & 3.839; & 39 & \\
\hline 900.475 .384 & 830.061.703; & 900.321 .035 & 900.512 .815 & 900.475 .243 & 900.382 .922 & 860.049.716; & $103 ;$ & 004 \\
\hline 900.503 .138 & 900.267 .576 & 890.111.847; & 830.142.615; & 800.089.017; & 830.111.973; & 7.107; & 800 & 55 \\
\hline & & & & & & & & \\
\hline 800.202.400; & 800.235.946; & 900.490 .530 & 830.027.712; & 800.102.384; & 900.451 .850 & 890.320.742; & 10.503 & 6.498 \\
\hline 900.702 .679 & 900.744 .734 & 800.241.053; & 900.199.705; & 830.145.560; & 900.488 .493 & 900.501.770; & 830.028.696; & 1.908 \\
\hline 813.012.783; & 830.124.909; & 830.126.910; & 830.095.024; & 900.329 .742 & 3.741; & 900.0 & 6.287; & 1.101; \\
\hline & & & & & & & & \\
\hline 65 & 800.050 & 900.222 .958 & 860.072.032; & 812.000.519; & .590 & .392 & 2.645; & 345 \\
\hline 900.383.695; & 800.012.335; & 860.049.602; & 892.001.701; & 830.505.329; & 900.019.950; & 900.055.341; & 900.235.133; & 1.490 \\
\hline 830.002.164; & 900.111 .968 & 900.177 .119 & 800.060.428; & 900.026 .402 & 900.071 .510 & 830.023.134; & 830.082.952; & 890.313.074 \\
\hline .126 & & & & 830.093.434; & 800.116.742; & 900.116 .324 & & \\
\hline 4.672 & 805.003. & 800.2 & 892.300.996; & 800.036.098; & 2.592; & 900.078 .272 & 34 & $.981 ;$ \\
\hline 900.354.220; & 900.095.399; & 800.145.838; & 800.030.718; & 900.426 .371 & 830.040.805; & 810.006.457; & 900.086.853; & 03.251 ; \\
\hline 900.674 .471 & 830.060.337; & 900.050 .106 & 900.097 .160 & 900.322 .758 & 804.007.858; & 805.030.001; & 830.506.018; & 900.210 .063 \\
\hline 900.394 .297 & 900.385 .737 & 805.015.467; & 860.502.016; & 830.057.592; & 900.064 .057 & 800.180.298; & 830.092.352; & 800.249.506 \\
\hline & 890 & & 800.183 & 900 & & 86 & 81; & 23 \\
\hline 830.058.578; & 800.120.390; & 817.000.611; & 890.703.111; & 860.519.810; & 900.465.905; & 830.115.084; & 890.317.768; & 830.508.750 \\
\hline 900.633 .376 & 892.002.955; & 860.072.036; & 900.492.179; & 890.101.357; & 800.137.477; & 900.265.816; & 830.139.501; & 800.031.913; \\
\hline 900.208.424; & 830.026.510; & 900.623 .247 & 800.038.165; & 890.921.681; & 900.007 .964 & 817.002.788; & 829.001.221; & 900.063 .764 \\
\hline & & & 830. & & & & & \\
\hline 900.342.373; & 900.610 .705 & 890.309.355; & 809.003.754; & 800.164.336; & 800.144.952; & 800.217.690; & 2.534; & 900.319.628; \\
\hline 860.351.301; & 900.534.970; & 900.367.790; & 810.006.207; & 900.488.129; & 900.540 .218 & 900.302 .621 & 800.081.826; & 900.189 .532 \\
\hline 860.518.132; & 800.066.621; & 860.037 .902 & 900.362 .793 & 830.041.061; & 900.065 .082 & 814.001.418; & 830.139.064; & 900.213 .535 \\
\hline 0.482 & 800.010 .567 & 830.145.038; & 830.140.793; & 800.129.475; & 800.188.197; & 900.082.958; & 800.108.287; & 900.155 .512 \\
\hline 357 & 860.005.620; & 900.138.191; & 800.023.452; & 900.051 .713 & 830.112.014; & 800.124.352; & 830.130.762; & 800.138.316 \\
\hline 830.512 .587 & 900.435.204; & 800.137.414; & 891.902.006; & 813.006.976; & 900.678 .026 & 900.311.228; & 820.001.601; & 900.069 .732 \\
\hline 860.451.696; & 830.064.925; & 811.014.294; & 860.030.991; & 800.019.946; & 830.039.072; & 830.506.018; & 890.401.905; & 810.001.845; \\
\hline 800.061.920; & 900.375 .643 & 890.940.445; & 800.129.166; & 800.041.077; & 802.008.945; & 900.263.879; & 860.450.997; & 836.000.694; \\
\hline & 800.207.622; & & 800.192 & 900.303.793; & 900 & & 84; & 609 \\
\hline 900.187.467; & 900.736.824; & 900.241 .902 & 900.205 .226 & 900.593 .346 & 900.384 .968 & 900.200.046; & 900.008 .927 & 900.516 .931 \\
\hline 800.019.719; & 900.680 .595 & 900.395.921; & 860.011.157; & 900.546 .904 & 900.462 .009 & 890.206.092; & 900.194.609; & 900.429.098; \\
\hline 900.027 .013 & 900.108 .758 & 900.430 .417 & 900.139.696; & 900.441 .837 & & 860.519.084; & & \\
\hline 830.1 & 900.635 .124 & 900.462 .379 & 900.212 .226 & 900.730 .505 & 900.615 .839 & 860.002.059; & 5.296 & 900.471 .060 \\
\hline & 900.386.019; & 900.626 .629 & 900.128 .071 & 900.532 .199 & 900.610 .392 & 800.195.041; & 830.104.423; & 830.038.798; \\
\hline 900.058.865; & 900.323.890; & 900.346.767; & 830.123.589; & 900.513 .173 & 900.471.263; & 860.534.169; & 800.207.018; & 801.003.325 \\
\hline 900.084 .338 & 900.494.363; & 900.077 .235 & 800.237.268; & 900.698 .517 & 900.275 .336 & 800.043.489; & 900.123 .400 & 813.002.158 \\
\hline 800.141.585; & 900.578 .979 & 900.457.954; & 811.002.366; & 900.749.983; & 900.208 .317 & 900.250.884; & 900.179 .772 & 900.168 .659 \\
\hline & 900.233 .028 ; & 900.606 .336 & 830.115.475; & 900.319.825; & 900.257 .048 & 800.206.926; & 900.028 .893 & 809.007.127; \\
\hline 900.118 .787 & 900.245.434; & 900.203.489; & 890.307.317; & 900.731.630; & 860.075 .613 & 890.930.926; & 832.007.381; & 900.130 .968 \\
\hline 900.095.996; & 900.696.706; & 900.474 .198 & 900.745 .572 & 900.649 .566 & 829.000.993; & 830.105 .696 & 830.120.596; & 830.138.650 \\
\hline 900.091 .812 & 900.339.779; & & 900.394.124; & 900.673.310; & 900.125 .827 & 860.502 .889 & 900.313 .210 & 900.067 .949 \\
\hline
\end{tabular}




\begin{tabular}{l|l|l|l|l|l|l|l|l}
$800.019 .792 ;$ & $900.576 .027 ;$ & $900.463 .145 ;$ & $900.518 .304 ;$ & $900.436 .755 ;$ & $860.510 .975 ;$ & $811.035 .938 ;$ & $900.052 .023 ;$ & $830.037 .572 ;$ \\
$830.118 .803 ;$ & $900.565 .443 ;$ & $900.665 .505 ;$ & $900.553 .673 ;$ & $900.223 .529 ;$ & & $900.135 .264 ;$ & $800.214 .850 ;$ & $900.137 .894 ;$ \\
$800.044 .150 ;$ & $900.563 .977 ;$ & $900.229 .513 ;$ & $900.617 .232 ;$ & $900.794 .198 ;$ & $900.624 .071 ;$ & $900.291 .137 ;$ & $900.482 .983 ;$ & $900.150 .921 ;$ \\
$830.114 .185 ;$ & $900.635 .730 ;$ & $802.024 .799 ;$ & $900.223 .793 ;$ & $900.523 .290 ;$ & 900.026 .823 & $900.451 .618 ;$ & $900.235 .350 ;$ &
\end{tabular}

Fuente: elaboración propia, con base en Benchmark, de BPR Asociados

Sales \& Credit Management (2017) y Rivera y Cuéllar (2018). 